\title{
What can we learn from the pervasive linkage of impulsivity and addictive behavior?
}

\section{Martin Zack*}

Neuroscience Research Department, Centre for Addiction and Mental Health, University of Toronto, Toronto, ON, Canada

${ }^{*}$ Correspondence: martin_zack@camh.net

\section{A commentary on}

Recreational drug use and impulsivity in a population of Canadian undergraduate drinkers

by Balodis et al., Front. Psychiatry 1:129 doi: 10.3389/fpsyt.2010.00129.

Trait impulsivity is consistently linked with pathological aspects of substance use. This study extends this linkage to an undergraduate population, in which scores on the Barratt Impulsivity scale were found to predict frequent binge drinking and poly-substance use. As the authors note, the acute and chronic effects of alcohol and drugs can promote impulsive behavior by impairing cortical executive functions. In this way, a preexisting tendency to impulsive behavior when coupled with exposure to psychoactive drugs could lead to a vicious cycle of frequent heavy use and a corresponding deterioration in executive functions. The authors observe that their cross-sectional data provide a basis to test this hypothesis in a longitudinal design. The data also raise a number of additional questions. First, what do the findings suggest about the role of screening and especially targeted prevention strategies in post-secondary institutions? More generally, what can we infer from the pervasive linkage of impulsivity and addictive behavior: Could trait impulsivity represent a "pro-dromal" state that mimics the deficits of non-impulsive individuals who have already transitioned to pathological levels of substance use? That is, in a functional sense, are impulsive individuals quasiaddicted from the outset?

Received: 11 September 2010; accepted: 13 September 2010; published online: 26 November 2010.

Citation: Zack M (2010) What can we learn from the pervasive linkage of impulsivity and addictive behavior? Front. Psychiatry 1:139. doi: 10.3389/fpsyt.2010.00139

This article was submitted to Frontiers in Impulsivity, Compulsivity and Behavioral Dyscontrol, a specialty of Frontiers in Psychiatry.

Copyright $\odot 2010$ Zack. This is an open-access article subject to an exclusive license agreement between the authors and the Frontiers Research Foundation, which permits unrestricted use, distribution, and reproduction in any medium, provided the original authors and source are credited. 